\title{
Leichtbau im Wandel
}

Wir erleben spannende Zeiten für den Leichtbau - oder erleben wir gar eine Zeitenwende? Die Bundesregierung bewertet Leichtbau zu Recht als Schlüsseltechnologie für den Wohlstand des Wirtschaftsstandorts Deutschland. Die Luftfahrt demonstriert seit vielen Jahren, wie die bewegte Masse von Hochtechnologiestrukturen massiv reduziert werden kann, ohne Abstriche bei Sicherheit und Wirtschaftlichkeit zu machen. Bedingt durch die Emissionsgesetze und die nahende Ära der Elektromobilität steht seit einigen Jahren ein weiterer, mächtiger Treiber der Leichtbautechnik auf dem Parkett: der Automobilbau. Und der wirft eine Frage auf, die so einfach klingt und dennoch so facettenreich ist: Wie etablieren wir Leichtbau für die Großserie? Die Frage beschränkt sich nicht mehr nur auf die Aufgabe, das Gewicht unserer Autos zu senken. Vielmehr geht es um den Auftrag, das beste Verhältnis von niedrigem Gewicht bei zugleich kostengünstiger und umweltverträglicher Produktionsweise zu erreichen - und dabei einen funktionieren Werkstoffkreislauf zu etablieren. Diese Fragen sind branchenübergreifend von großem Interesse, denn auch Maschinenbau, Schiene, Schiffbau und Windenergie verlangen nach leichten, kostengünstigen und umweltverträglichen Strukturen.

Dieser Bedeutung stellen wir uns als Leichtbauer. Es ist uns bewusst, dass Leichtbau mittlerweile über die Substitution von Werkstoffen auf Komponentenebene hinausgeht. Leichtbau im Jahre
2017 bedeutet, Potenzial in einer gesamten Struktur, einem gesamten Produkt, einem gesamten Fahrzeug auszuschöpfen. Wir stehen vor dem Zeitalter des Systemleichtbaus: An die Stelle der Optimierung von bekannten Produkten rückt die Entwicklung neuartiger, gewichtsoptimierter Systeme. Mit der Diskussion über Gewichtseinsparungen durch 800-V-anstelle von 400-V-Netzen in künftigen Elektroautos bewegen wir uns bereits jenseits der Frage nach dem richtigen Werkstoff.

Unter meinem Vorgänger Ulrich Knorra hat sich die lightweight.design zu einem zentralen Organ für den fachlichen Austausch unter Entwicklern und Anwendern von Leichtbautechniken entwickelt. Ich freue mich, diese Tradition nun fortführen zu dürfen und Ihnen alle zwei Monate eine Auswahl der neuesten Entwicklungen und relevantesten Themen präsentieren zu dürfen. Eines steht dabei jedoch fest: Den größten Anteil am Erfolg dieses Hefts haben Sie!

Sie sind die Experten für die vielfältigen Techniken des Leichtbaus. Sie forschen an neuen Verfahren und entwickeln neue Produkte für den Markt. Die lightweight.design verstehe ich dabei als Ihr Forum, dessen Aufgabe es ist, den Austausch über technische und wissenschaftliche Neuheiten unter Ihnen zu fördern. Deswegen lade ich Sie herzlich ein, am Erfolg des Hefts mitzuwirken. Ich nehme jederzeit gerne Themenvorschläge für Fachbeiträge von Ihnen entgegen.

Auf eine gute Zusammenarbeit! 